\section{Corrección de una maloclusión Clase III de Angle en paciente adulto con la técnica Multiloop Edgewise Archwire con un control post tratamiento de 1 año}

\author{
Correction of a Class III Angle \\ malocclusion in an adult patient with the \\ Edgewise Archwire Multiloop technique \\ with a 1-year post-treatment control
}

\section{Caso Clínico}

Mariana Valdez Galdos ${ }^{1, a}$, Armando Fernandez Rivas ${ }^{1, b}$

${ }^{1}$ Universidad Peruana de Ciencias Aplicadas, Facultad de Odontología. Lima, Perú.

a Cirujano Dentista.

${ }^{\mathrm{b}}$ Especialista en Ortodoncia y Ortopedia Maxilar.

\section{Correspondencia:}

Mariana Valdez Galdos: marianavg85@gmail.com Urb. Quinta Samay A-2 Cayma, Arequipa - Perú. ORCID: 0000-0001-6257-6441

\section{Coautores:}

Armando Fernández Rivas

amfernandezr@gmail.com

\section{Editora:}

Sandra Patricia Palomino-Gómez

Universidad Nacional Mayor de San Marcos, Perú.

Conflictos de intereses: sin conflicto de intereses.

Fuente de financiamiento: autofinanciamiento.

Recibido: 15/09/19

Aceptado: $25 / 11 / 19$

Publicado: 24/02/20

\begin{abstract}
Resumen
Tradicionalmente el tratamiento de la compensación, el camuflaje, de las maloclusiones de Clase III, ha estado orientado a la extracción de primeros premolares inferiores, distalización de caninos y lingualización de incisivos inferiores con la finalidad de obtener un overbite y overjet aceptables. El propósito de este reporte es describir el diagnóstico y plan de tratamiento de una maloclusión Clase III, en un paciente adulto, soportado por una revisión bibliográfica. El caso fue tratado con el sistema Multiloop Edgewise Arch Wire $(M E A W)$ y se realizaron exodoncias de terceros molares inferiores. El sistema $M E A W$ utiliza arcos con dobleces colocados en los espacios dentarios interproximales, desde la parte distal de los incisivos laterales hasta la última pieza en el sector posterior, al cual se le realizan ciertas activaciones según la maloclusión. Se puede concluir, que con este sistema es posible compensar los casos de Clase III mediante la reconstrucción del plano oclusal, evitando así el realizar exodoncias de premolares inferiores. Además, controlando el efecto de lingualización de los incisivos inferiores se genera un correcto overjet y overbite y relaciones caninas Clase I.
\end{abstract}

Palabras clave: Ortodoncia; Aparatos ortodóncicos fijos; Clase III de Angle (fuente: DeCS BIREME).

\begin{abstract}
The treatment of compensation or camouflage of Class III malocclusions was traditionally oriented to the extraction of first lower premolars, canine distalization and lingualization of lower incisors in order to obtain an acceptable overbite and overjet. The purpose of this report is to describe the diagnosis and treatment plan of a Class III malocclusion in an adult patient, supported by a literature review. The case was treated with the Multiloop Edgewise Arch Wire (MEAW) system and lower third molars were extracted. The MEAW system uses arches with loops placed in the interproximal dental spaces, from the distal part of the lateral incisors to the last tooth in the posterior area, to which certain activations are performed according to the malocclusion. It can be concluded that with this system the compensation of Class III cases is possible through the reconstruction of the occlusal plane and avoiding the extraction of lower premolars. Also, controlling the
\end{abstract}


lingualization effect of the lower incisors a correct overjet and overbite and Class I canine relations can be achieved.

Keywords: Orthodontics; Fixed orthodontic appliances; Class III of Angle (source: MeSH NLM).

\section{Introducción}

La maloclusión de Clase III, comprende un conjunto de características faciales y esqueléticas, dentro de las cuales podemos encontrar una maxila retruida, denominada retrognatismo maxilar, una mandíbula protruida o definida como prognatismo mandibular o una combinación de ambas, que se integran para expresar de manera particular las relaciones sagitales maxilo-mandibulares. Además, la desarmonía esquelética se encuentra acompañada de características dentarias particulares según la compensación que el equilibrio entre musculatura, huesos y dientes, puedan condicionar.

Según Ravindra Nanda, el manejo de este tipo de maloclusiones lo convierte en "uno de los tratamientos más desafiantes en ortodoncia" dado que el paciente de Clase III o considerado también por el autor como prognata puede definirse como poseedor de "una deformidad facial esquelética caracterizada por una proyección mandibular hacia adelante con respecto a la base craneal y/o maxilar" ${ }^{1}$.

La maloclusión de Clase III tiene una gran amplitud de problemas lo que conduce a un diágnóstico desafiante, pues varía según la gravedad de los problemas dentoalveolares, el posible desplazamiento funcional anterior de la mandíbula, hasta problemas esqueléticos verdaderos con discrepancias maxilomandibulares graves ${ }^{2-5}$.

Con la incorporación de la cefalometría en las herramientas del diagnóstico, es posible realizar un estudio más específico de las relaciones esqueléticas y dentoalveolares. Los diversos análisis cefalométricos propuestos permiten una determinación más precisa de las alteraciones en tamaño y posición de las estructuras anatómicas. En el caso de las maloclusiones de Clase III el estudio cefalométrico nos proporciona información característica y de acuerdo a lo propuesto por Guyer et al., estos pacientes pueden presentar una longitud de la base craneal posterior (S-Ba) significativamente más larga, un maxilar generalmente en posición retrusiva, con una longitud efectiva significativamente más corta, una mandíbula prognática vista desde una posición sagital (3-6 mm más largo que una Clase I), con un ángulo gonial característicamente más obtuso y posicionado anteriormente. El ángulo del plano mandibular tiende a ser mayor en la Clase III. Suele encontrarse una mayor altura vertical inferior con una compensación dentaria en la que los incisivos maxilares se encuentran significativamente más protruidos ${ }^{6}$.

Uno de los parámetros más importantes en el diagnóstico de las maloclusiones de Clase III, se encuentra en el análisis del plano oclusal, que se constituye como el componente más influyente de la dimensión vertical de la cara inferior. De acuerdo a lo propuesto por el profesor Sadao Sato, "la posición vertical de los dientes posteriores no es estable durante el crecimiento, en las Clases III de ángulo alto y en las mordidas abiertas, la erupción continua de las molares ocurre no solamente durante el crecimiento sino también durante el periodo post puberal. En este sentido, la genética puede no ser la única razón de este tipo de maloclusión. Por el contrario, la continua erupción de los segundos y terceros molares en un espacio limitado debe ser el principal factor contribuyente. El desarrollo de tales maloclusiones debe considerarse como un efecto de discrepancia o apiñamiento posterior" ${ }^{7}$.

La discrepancia posterior aumenta la probabilidad de impactación de los terceros molares y una vez que se produce su impactación puede producirse un efecto llamado "squeezing-out", causando una erupción excesiva de los dientes adyacentes, aplanar el plano oclusal posterior y ocasionar un aumento en la dimensión vertical posterior. Los molares sobre erupcionados producen interferencias oclusales que actúan como un punto de apoyo que causa una adaptación de la mandíbula, ubicándola hacia adelante permitiendo una subluxación de los cóndilos y una remodelación activa del cartílago condilar. El resultado de esta alteración es un prognatismo mandibular ${ }^{8-11}$.

El tratamiento descrito en este reporte fue realizado con el sistema Multiloop Edgewise Arch Wire (MEAW), que fuera propuesto por el Dr. Kim, concebido inicialmente para el tratamiento de las mordidas abiertas. Este sistema comprende la elaboración de arcos de Elgiloy azul 0,016 " x 0,022 " con loops horizontales colocados en los espacios interproximales desde la parte distal de los incisivos laterales hasta mesial de las últimas piezas posteriores y requieren de ciertas activaciones según la maloclusión ${ }^{12-14}$.

El propósito de este reporte es proponer el uso del sistema $M E A W$ como alternativa eficiente en el tratamiento de camuflaje de las maloclusiones de Clase III, evitando las extracciones de premolares.

\section{Reporte del caso}

Este reporte presenta el caso de un paciente adulto de sexo masculino con relación esquelética de Clase III, por deficiencia maxilar acompañada de biprotrusión dentaria y mordida borde a borde, maloclusión de Angle de Clase III. Este paciente fue tratado satisfactoriamente logrando los objetivos de establecer relaciones molares y caninas de Clase I, obtener una oclusión funcional y balanceada, y lograr una mejor armonía facial. 
Diagnóstico. Paciente de sexo masculino de 27 años de edad acude a consulta con el motivo: "Quiero mejorar la posición de mis dientes anteriores y no tener dificultad para desgarrar los alimentos con los dientes anteriores".

En el examen extraoral se observó que el paciente tiene un biotipo dolicofacial, un perfil convexo y biprotruso. El examen intraoral reveló relaciones molares y caninas Clase III, mordida borde a borde, arcadas dentarias grandes (Figura 1).

En el análisis cefalométrico inicial se obtuvieron los siguientes datos: relación esquelética Clase III según Steiner, relación intermaxilar Clase III según Wits, altura facial aumentada, longitud de la mandíbula aumentada e hiperdivergente, incisivos superiores e inferiores vestibularizados y protruidos (Figura 2). En la radiografía panorámica se observa la presencia de terceros molares inferiores en oclusión (Figura 3). En el análisis de modelos iniciales se obtuvo una discrepancia dentoalveolar de - 2,5 mm maxilar, y - 4,1 mm mandibular. No presenta problemas transversales (Figura 4).

Tratamiento. Habiéndose instalado la aparatología ortodóntica con prescripción Roth, ranura 0,022”x 0,028” (GAC), se llevó a cabo la primera fase de alineamiento y nivelación con arcos NiTi 0,014 " y 0,018 ", y luego arco NiTi 0,017 " x 0,025 ". Debido a compromisos laborales del paciente, recién finalizada esta fase, se realizó la exodoncia de los terceros molares inferiores, con el objetivo de eliminar la discrepancia posterior. Según la literatura, se recomienda que los terceros molares superiores e inferiores deben ser extraídos previamente al inicio del tratamiento ${ }^{15}$.

En la segunda fase se tomó impresiones al paciente para la obtención de modelos en yeso en los que se confeccionaron los arcos $M E A W$ con alambre Elgiloy azul 0,017" x 0,025 ", con la finalidad de lograr un mejor control del torque.

Las razones para realizar los dobleces de los loops horizontales en el arco son las siguientes: disminuir la relación de carga/deflexión, proporcionando una fuerza de ortodoncia baja pero continua sobre los dientes. El loop horizontal permite un control más fácil del movimiento para cada diente, facilita la alineación y la intrusión del diente supra erupcionado, así como un sencillo ajuste del torque. Una vez conformados los arcos e incorporadas las activaciones de estos, es necesaria la ayuda de los elásticos intermaxilares, para obtener una correcta intercuspidación y la reconstrucción del plano oclusal ${ }^{16}$.
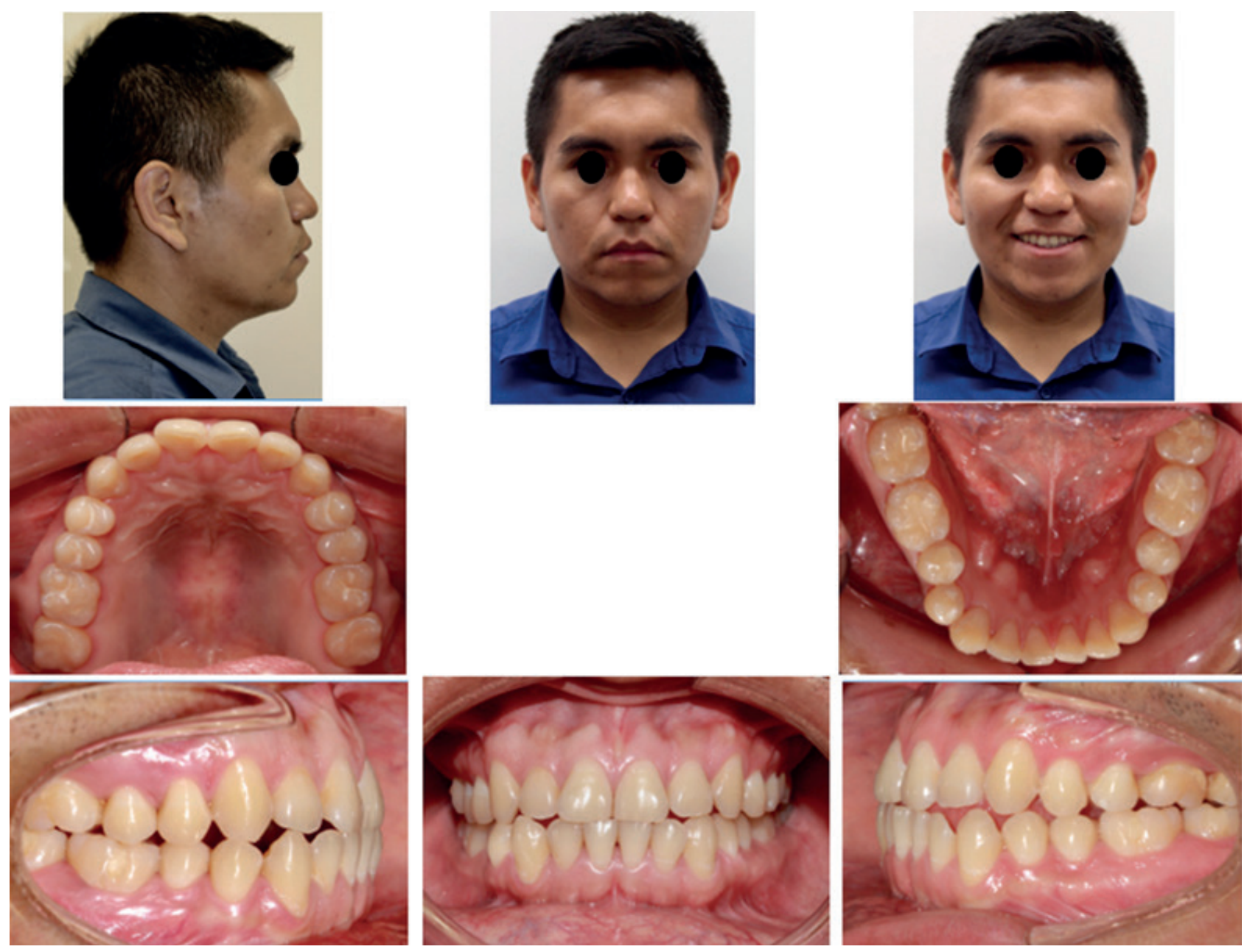

Figura 1. Fotografías intraorales y extraorales iniciales 


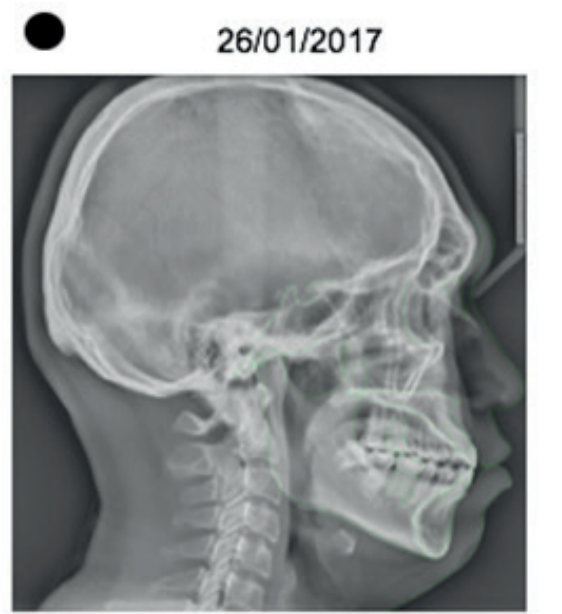

\begin{tabular}{|c|c|c|c|c|}
\hline & & Norma & Incial & Final \\
\hline & $\mathrm{SN}$ & $70(+-3)$ & 70 & 70 \\
\hline & SNA & $82(+-3)$ & 79 & 79 \\
\hline & SNB & $80(+-3)$ & 80 & 80 \\
\hline & ANB & $2(+-3)$ & -2 & -2 \\
\hline & A-Nperp & $0 / 1$ & $-5,4$ & $-4,4$ \\
\hline & Pg-Nperp & $-8 a-6 /-2 a+4$ & -10 & $-8,1$ \\
\hline & AFAinf & $60-62$ & 70,6 & 68 \\
\hline $18 / 10 / 2018$ & F Ejey & $60(+-3)$ & 67 & 66 \\
\hline & 1-NA & $4 \mathrm{~mm}$ & 9,1 & 7,8 \\
\hline & 1.NA & $22^{\circ}$ & 30 & 28 \\
\hline & 1-NB & $4 \mathrm{~mm}$ & 7,5 & 6 \\
\hline & 1.NB & $25^{\circ}$ & 31 & 28 \\
\hline & 1.1 & 130 & 118 & 124 \\
\hline & SNV-UL & $3-5$ & 5,6 & 6,9 \\
\hline & SNV-LL & $0-2$ & 6,0 & 6,1 \\
\hline & SNV-Pg' & $-4 a 0$ & $-5,2$ & $-4,4$ \\
\hline
\end{tabular}

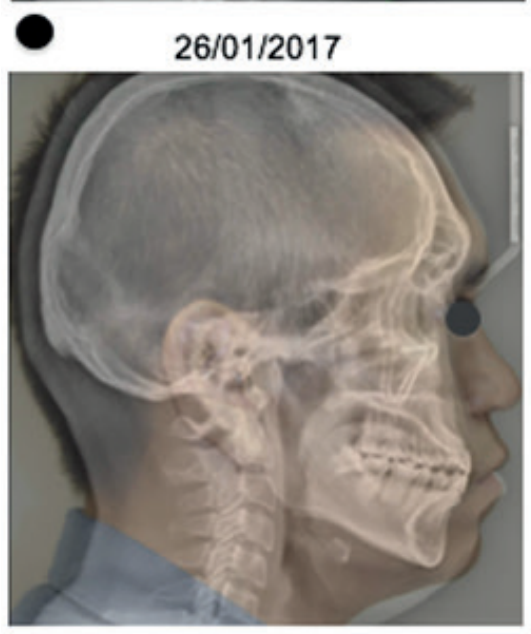

Figura 2. Radiografía lateral inicial y superposición de cefalometría con fotografía de perfil inicial

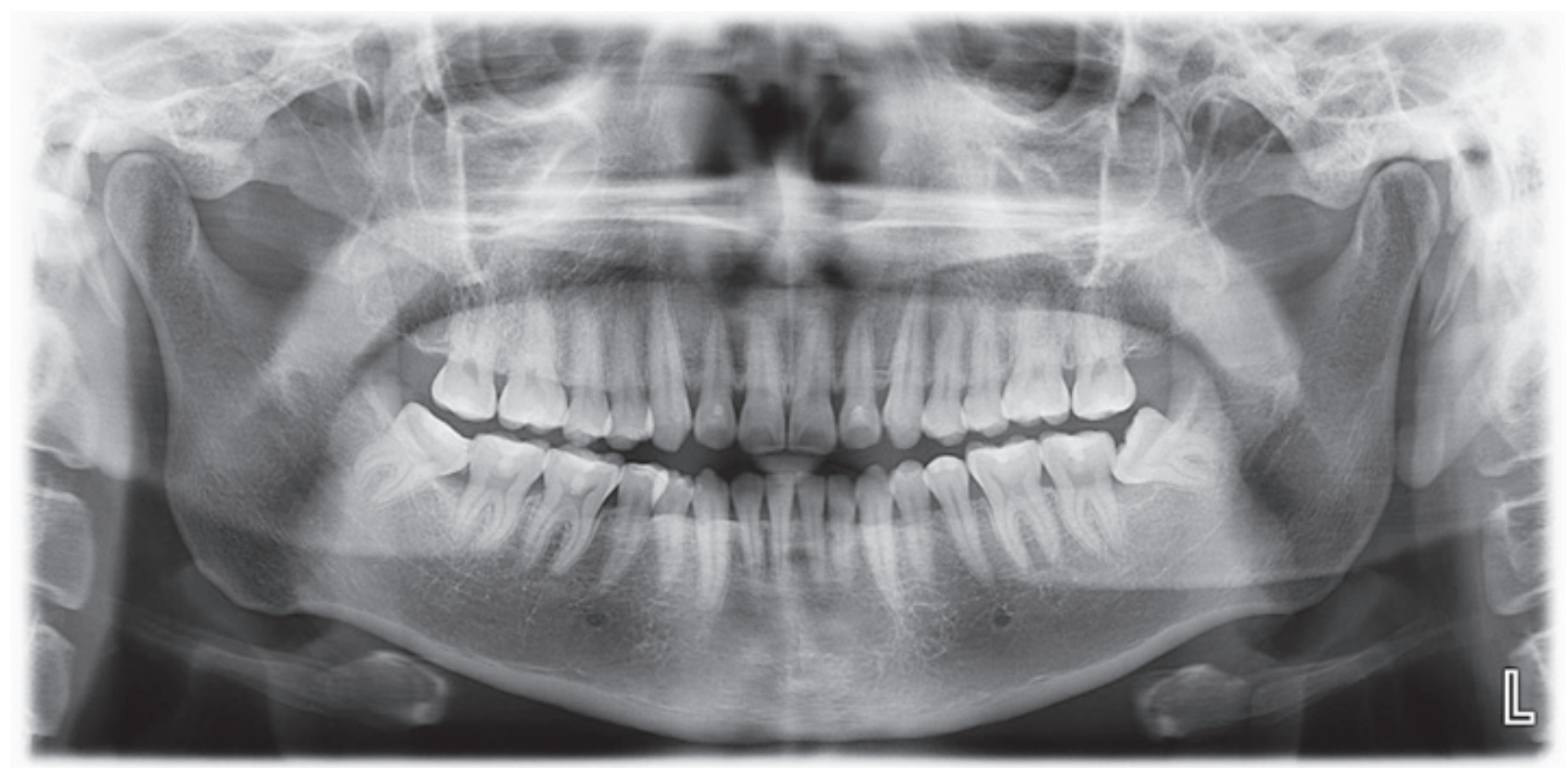

Figura 3. Radiografía panorámica inicial 


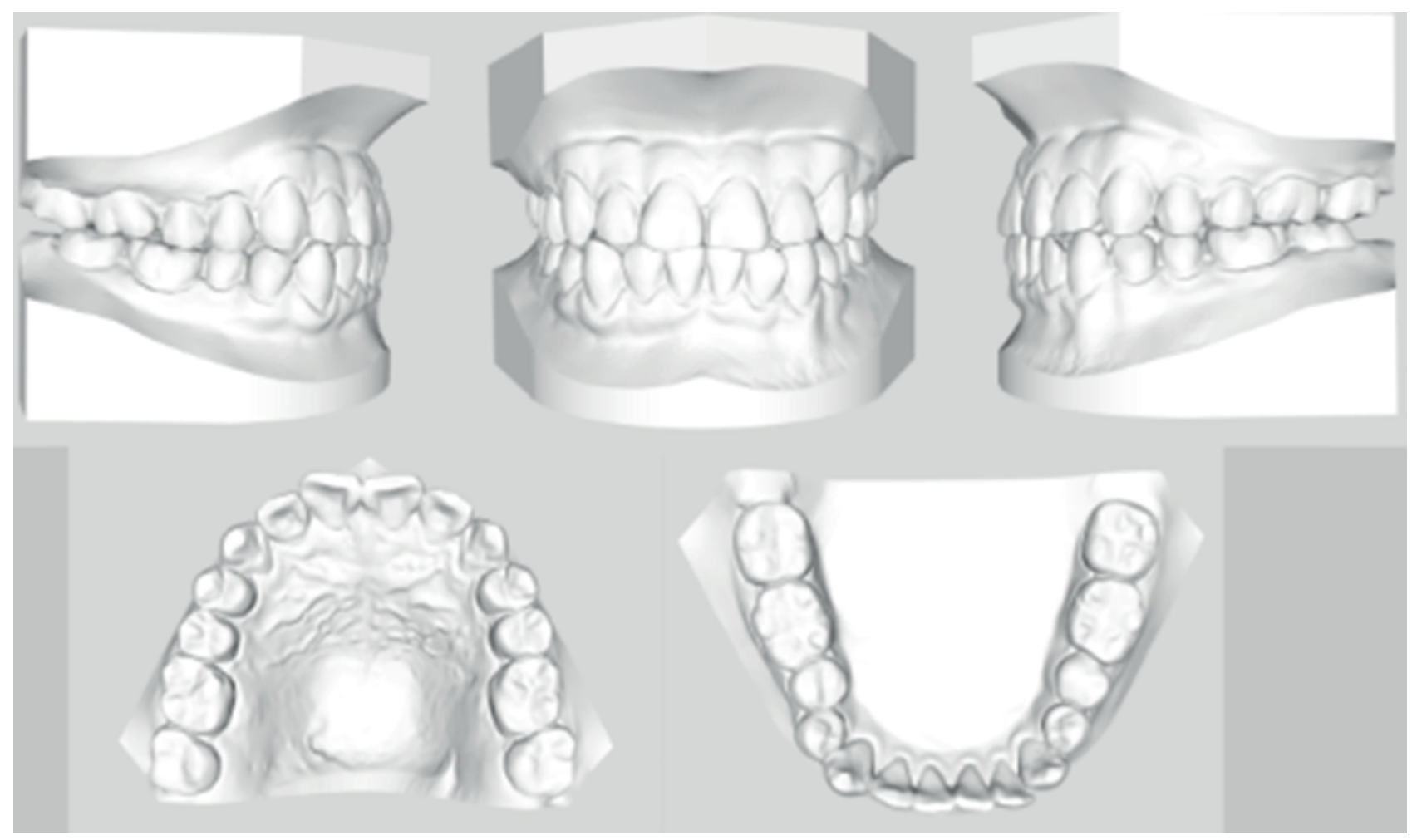

Figura 4. Registro de modelos digitales iniciales

En estos arcos conformados se realizaron las siguientes activaciones, previo a la instalación en boca: la primera activación consistió en dobleces de Tip back con curva acentuada en el arco superior y dobleces de Tip back con curva invertida en el arco inferior, esta activación se realiza de distal de canino a segundo molar. Se aplican dobleces de tip-back para obtener los cambios de angulación requeridos ${ }^{16}$.

Los arcos activados de la manera descrita generarían una mordida abierta, por lo que se indicaron elásticos verticales del primer loop superior a primer loop inferior en ambos lados para controlar este efecto indeseado.

En la segunda activación se confeccionó dobleces de Step up a nivel de segundos y primeros premolares inferiores, y dobleces de Step down a nivel de segundos y primeros premolares ${ }^{16}$. Adicionalmente, se indicó el uso de elásticos intermaxilares de Clase III. La tercera activación consistió en dobleces de Step down en el sector anterosuperior para aumentar el overbite y se corrigió los torques de los incisivos superiores.

Una vez establecidas las relaciones molares y caninas correctas se procedió a la desactivación de los dobleces de Tip back, se aplanó la curva de spee superior e inferior, y se indicó el uso de elásticos intermaxilares verticales y Clase III ${ }^{16}$. Para la quinta activación se retiró el uso de elásticos intermaxilares Clase III, y se continuó sólo con elásticos verticales. Además, se desactivaron los dobleces de Step up y Step down en premolares ${ }^{16}$.

Por último, en la fase de acabado y finalización, se colocaron arcos de TMA 0,017 x 0,025 sin loops, incorporándose los siguientes dobleces: ligero torque negativo en incisivos inferiores, y doblez de rotación e intrusión en la pieza 47. Los avances del tratamiento se observan en la Figura 5.

Los resultados del tratamiento cuya duración fue de 18 meses se evidencian en las figuras 6-8. A través de las superposiciones se observan los cambios dentoalveolares y de tejido blando (Figura 9). En la evaluación dentaria del maxilar superior se observó la extrusión y verticalización del primer molar superior, vestibularización de incisivos centrales superiores. En la relación vertical de la maxila se observa una ligera rotación de la maxila en sentido antihorario. La relación horizontal de la maxila no mostró cambios. En la evaluación de la mandíbula se observó un reacomodo de esta en sentido antihorario mostrando una ligera proyección del mentón hacia adelante. En la evaluación de los cambios dentarios de la arcada inferior se observó distalización y distoinclinación del primer molar e incisivo inferior, también se observaron cambios favorables a nivel del labio inferior, mostrando una mejor relación con el superior y una mejora significativa de la apariencia estética del paciente.

En la Figura 10 se pueden observar las imágenes a un año de control post tratamiento, que muestran una relativa estabilidad del caso.

\section{Discusión}

Los doctores McLaughlin, Bennett, y Trevisi en una referencia al manejo clásico del camuflaje de Clase III, manifiestan que consiste en una compensación dental que se consigue con inclinación anterior de los incisivos superiores y retroinclinación de los incisivos inferiores, 

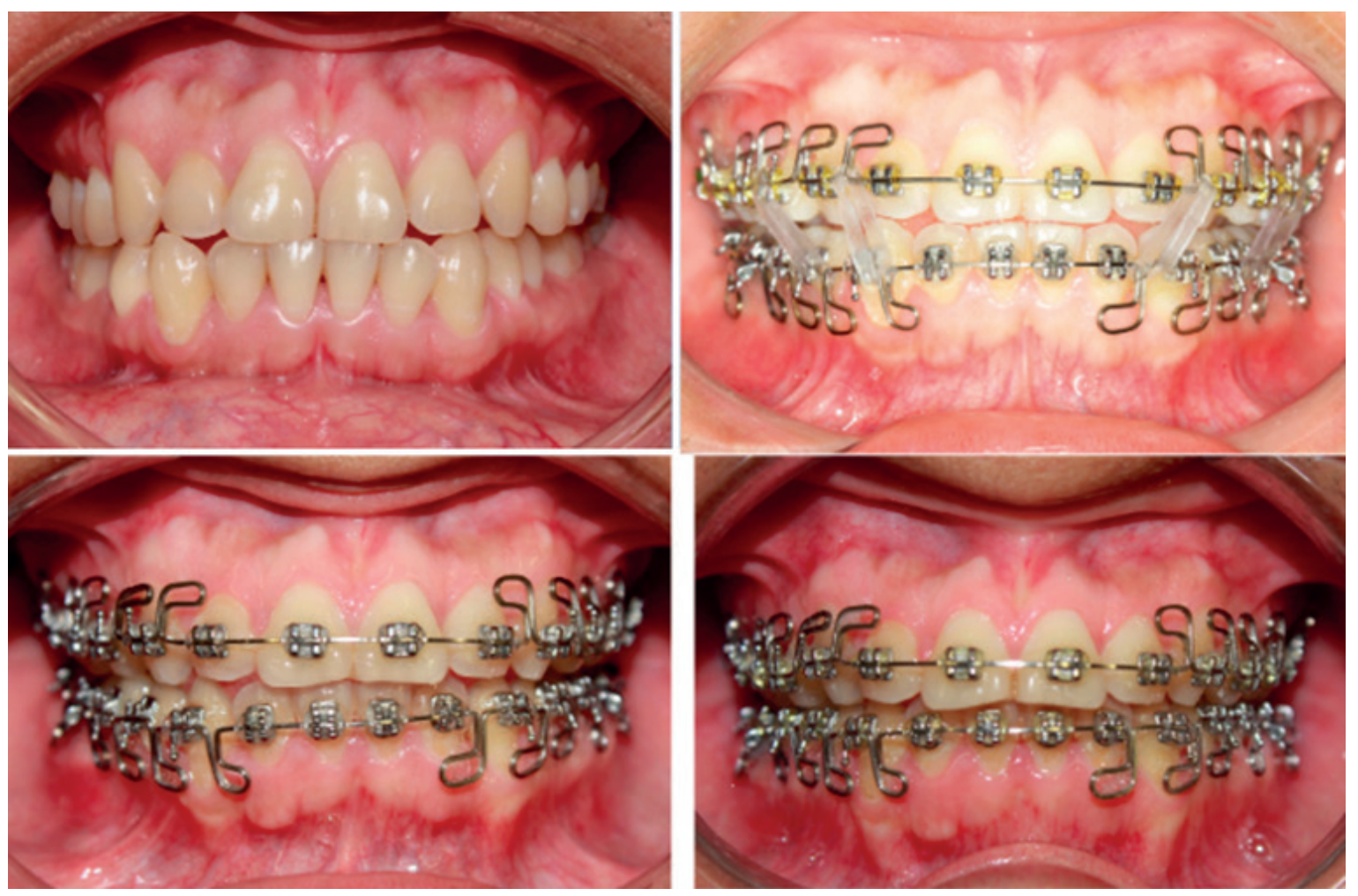

Figura 5. Avance fotográfico vista frontal
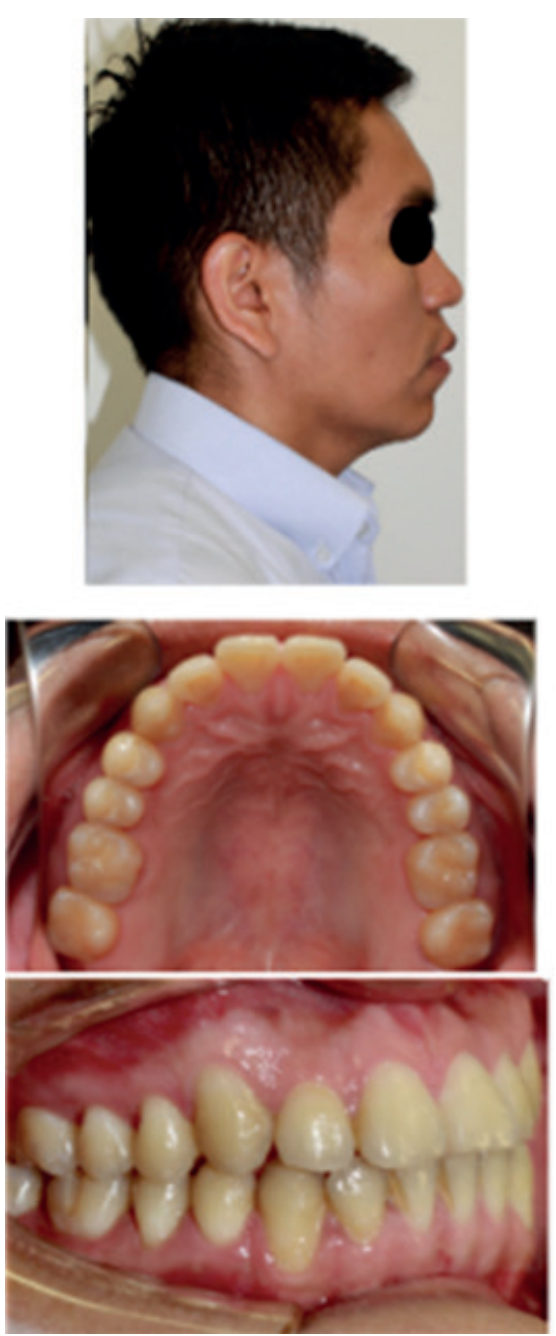
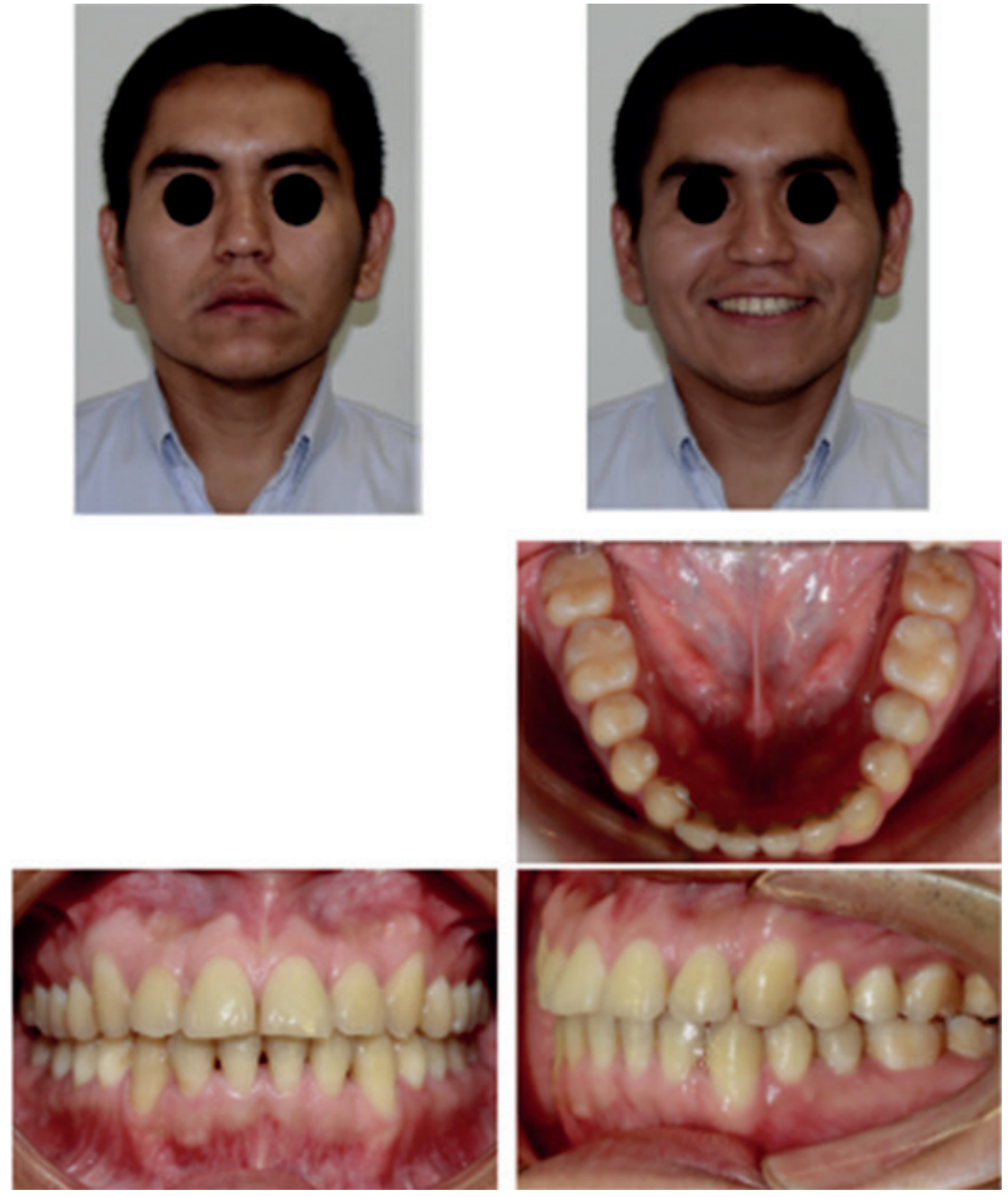

Figura 6. Fotografías intraorales y extraorales finales 


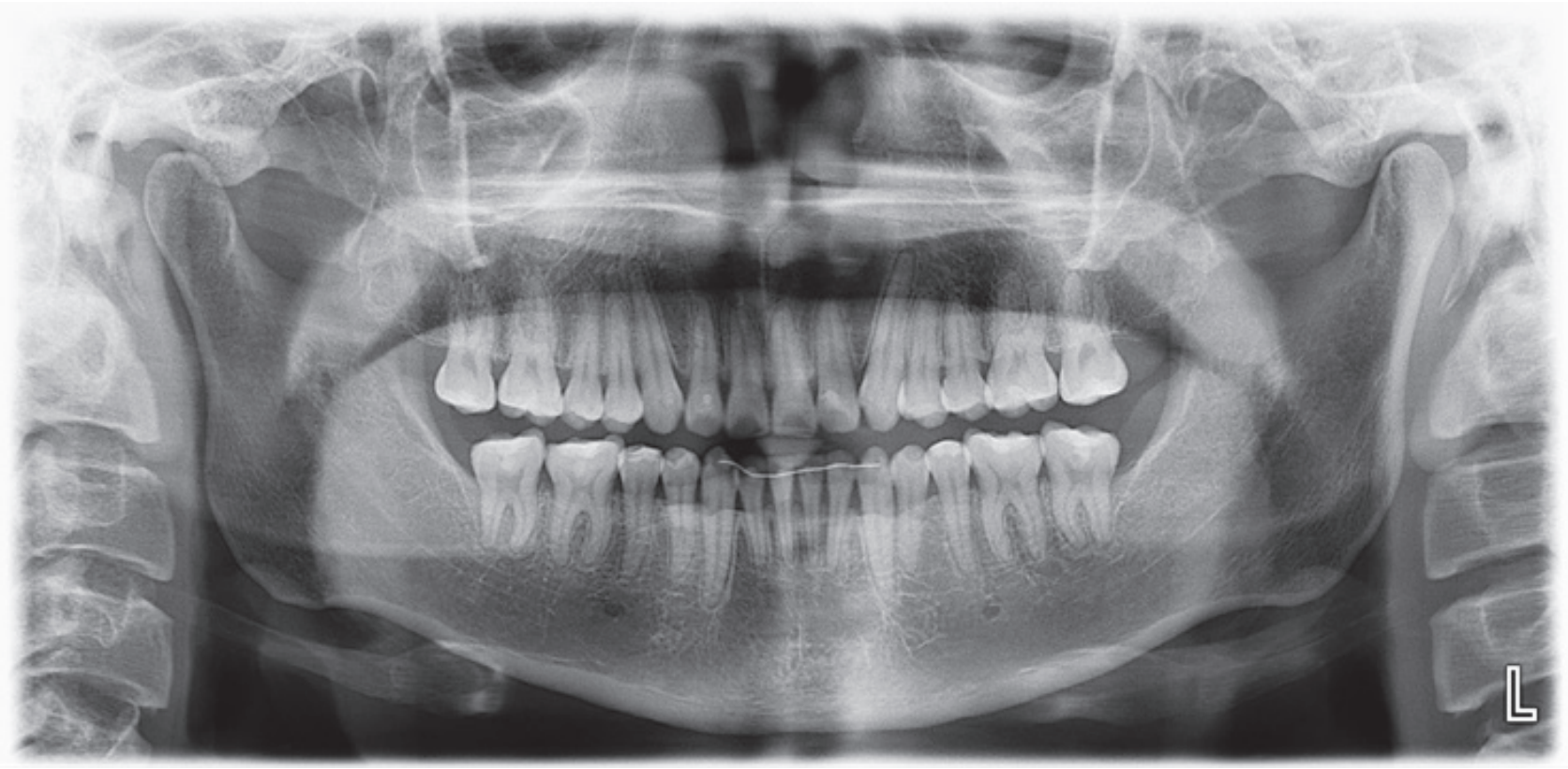

Figura 7. Radiografía panorámica final
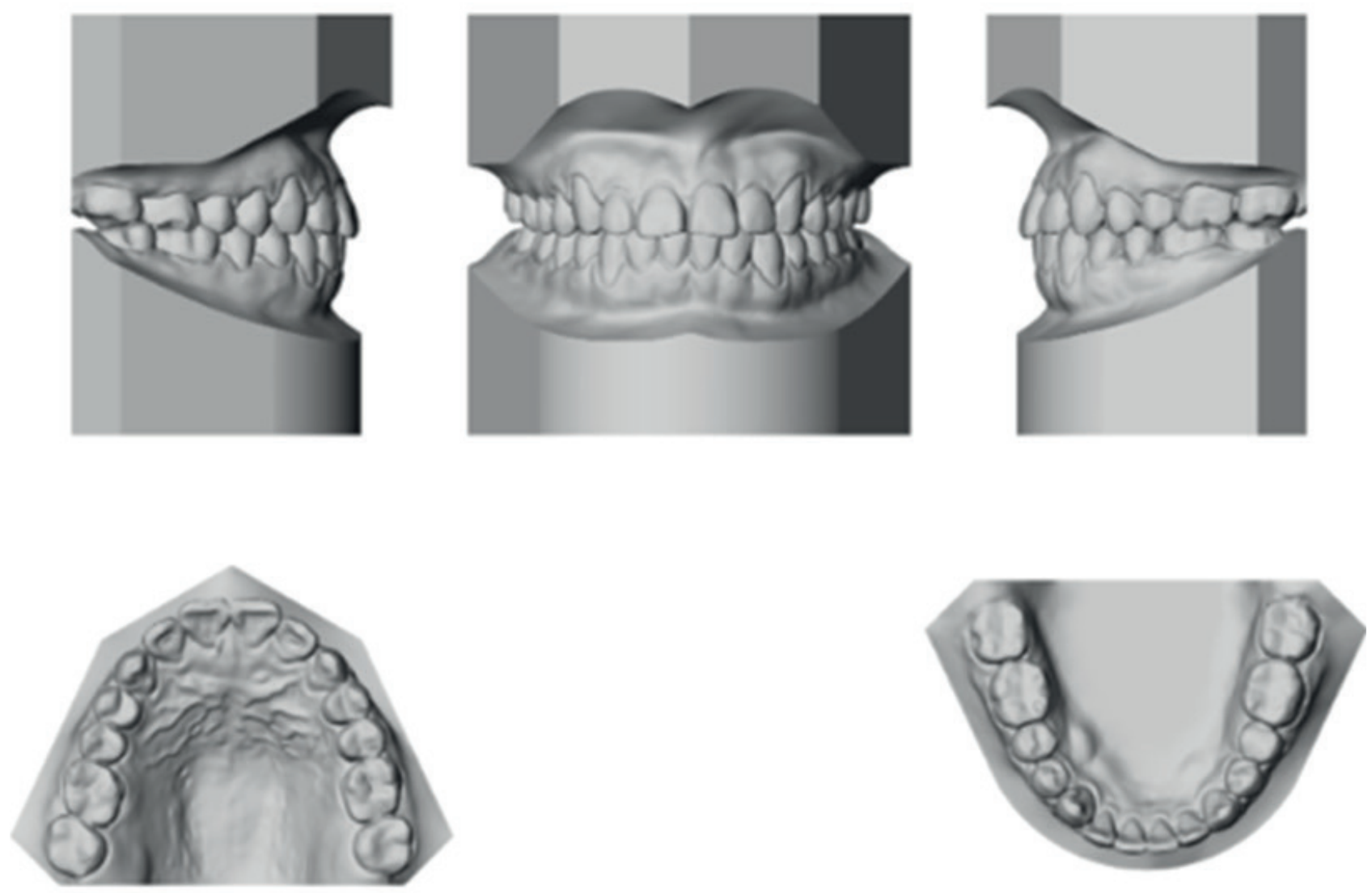

Figura 8. Registro de modelos digitales finales 

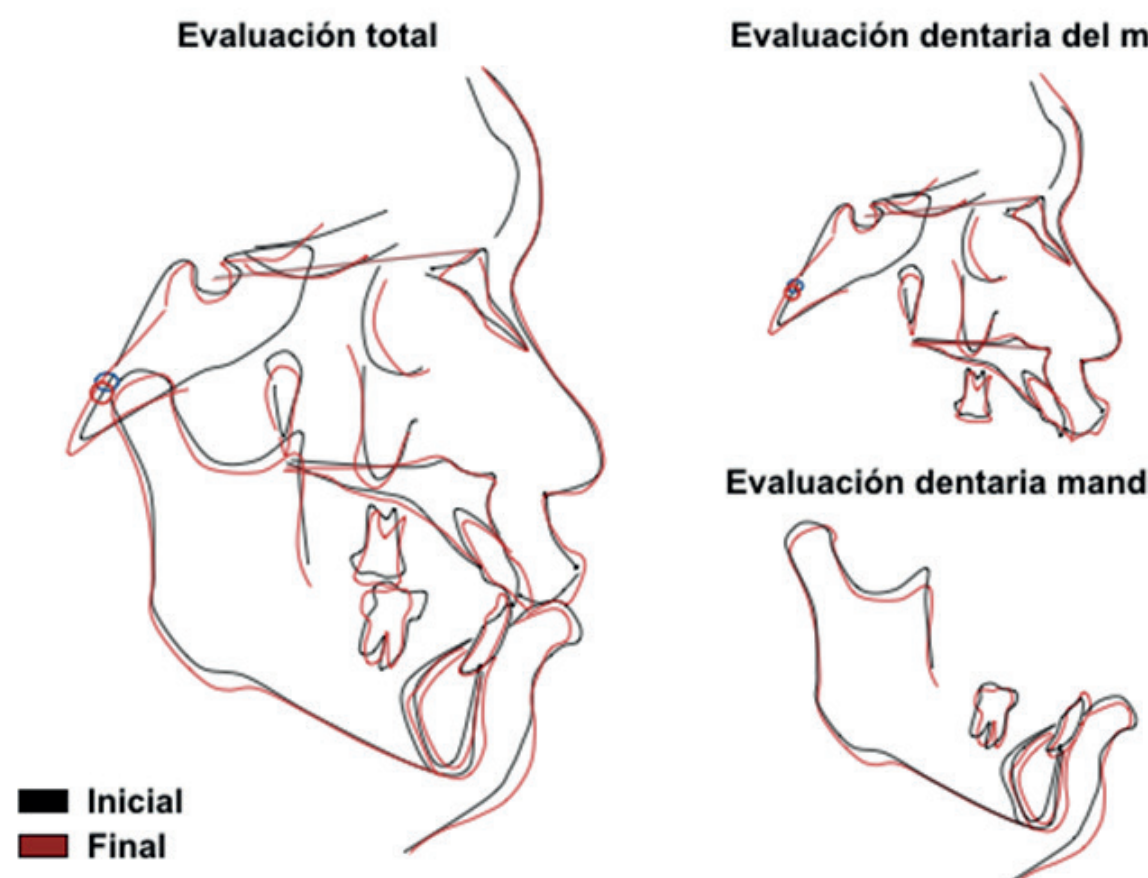

Evaluación dentaria mandibular

Figura 9. Superposiciones de cefalometría inicial y final
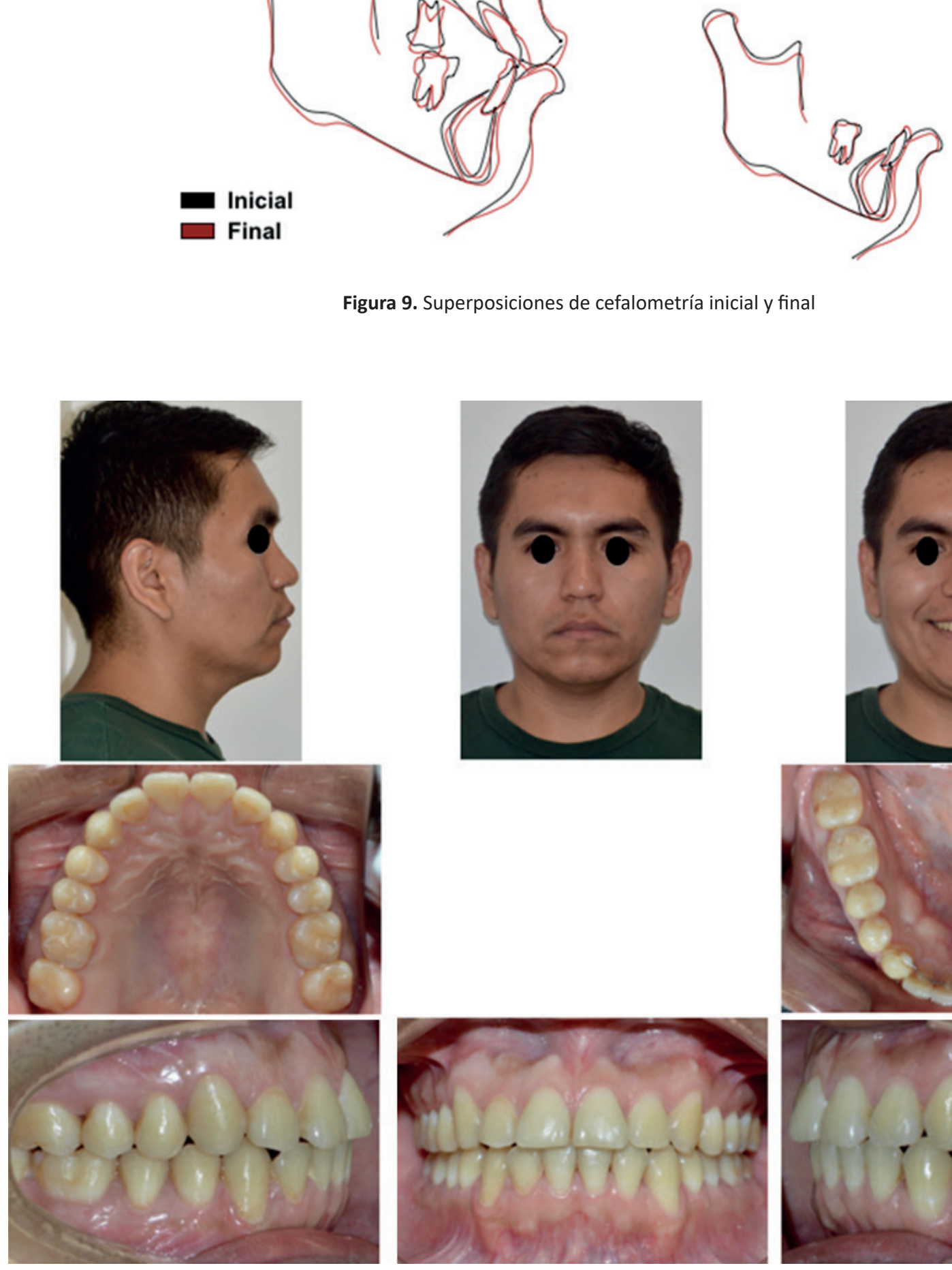

Figura 10. Fotografías de control 1 año post tratamiento 
acompañado del uso de elásticos de clase III, pero teniendo en cuenta en primer lugar que existen límites al movimiento mesial de los incisivos superiores, por lo que debemos evitar su proinclinación excesiva, de lo contrario se obtendría una apariencia no estética además de una función inadecuada. Los autores mencionados proponen como regla general que se debe evitar proinclinar los incisivos superiores más allá de los $120^{\circ}$ respecto al plano maxilar, a pesar de las variaciones individuales que puedan presentarse ${ }^{17}$.

Varios reportes mencionan que una estrategia común del tratamiento de camuflaje de ortodoncia es el uso de elásticos intermaxilares de clase III para corregir la discrepancia sagital. Los elásticos de clase III dan como resultado un movimiento mesial de la dentición superior y un movimiento distal de la dentición inferior con la proclinación de la dentición superior y la retroclinación de la dentición inferior. También inducen la extrusión de los molares superiores y los incisivos inferiores, lo que da como resultado una rotación en sentido antihorario del plano oclusal y un aumento de la altura facial. La posición e inclinación de los incisivos superiores y la rotación plano oclusal son componentes importantes de la estética facial y de la sonrisa. Estos cambios podrían darnos resultados estéticos desfavorables ${ }^{18-21}$.

Se puede asumir que el manejo biomecánico de un tratamiento de camuflaje de un caso de Clase III con biprotrusión no podría ser realizado sin las extracciones de premolares inferiores y la inclinación labial de los incisivos superiores, sin embargo la reconstrucción del plano oclusal propuesta con la filosofía de tratamiento $M E A W$, permite obtener resultados satisfactorios en el manejo de este tipo de tratamientos evitando los efectos no deseados que conllevan las extracciones propuestas en los antecedentes. Podemos así pasar de una maloclusion de Clase III a una oclusión funcional y balanceada de Clase I con un overbite y overjet adecuados manteniendo la totalidad de piezas dentarias de una formula de 28 dientes realizando únicamente las extracciones de las terceras molares. Esto es posible debido a que la incorporación de la filosofía $M E A W$ genera los siguientes cambios descritos por el Dr. Sadao Sato en la literatura: Las piezas posteriores son intruídas y verticalizadas de tal manera que el plano oclusal puede ser reconstruido y la mandíbula reposicionada posteriormente. Y la maxila puede prolongarse hacia abajo y hacia adelante ${ }^{16}$. Los premolares y molares pueden distoinclinarse simultáneamente por medio del Tip-back. Según Kim (1987), la magnitud de un Tip-back es de 3-5 grados, y esto puede aumentarse si es necesario en el tratamiento ${ }^{23}$.

Es importante mencionar que los arcos $M E A W$ funcionan bajo tres mecanismos básicos:

a) Verticalización mesiodistal de los dientes para mejorar la dimensión vertical y el plano oclusal y ganar espacio mesiodistal para la dentición. b) Corrección de las rotaciones mesiales de los dientes, generando expansión en el arco para ganar espacio horizontal para la dentición. c) Verticalización bucolingual de los dientes para mejorar la dimensión vertical y establecer una guía adecuada y una oclusión funcional. El sustento sobre el cual se toma la decisión de no extraer premolares en este tipo de casos de compensación se basa en la importancia que tiene el primer premolar como punto de fulcro en la reconstrucción del plano oclusal. El primer premolar al encontrarse en una posición anteroposterior en el centro del arco se convierte en un punto pivotante en el control vertical, debido a que se encuentra a la mitad del camino entre los dientes que componen la discrepancia posterior y la zona de la musculatura de la matriz funcional. Es un diente menos afectado por las actividades musculares masticatorias y es el diente posterior más distante de la ATM lo cual lo torna muy efectivo para establecer la posición mandibular ${ }^{23}$.

Chang et al. (2004) sostienen, que la principal ventaja del sistema $M E A W$ es generar una menor discrepancia en la cantidad de desplazamiento de los dientes y una distribución relativamente más uniforme del estrés en toda la dentición que un simple arco ideal.

Esto significa que el movimiento distal en masa de toda la dentición mandibular podría realizarse más fácilmente con la mecánica $M E A W$. La explicación de este fenómeno se debe a la relación de deflexión de carga relativamente más baja en los segmentos posteriores de los arcos multiansas, que se origina en la longitud horizontal más larga de los L-loops (Yang et al., 2001). Además, Jin et al. (2004) reportaron que el cambio principal después del tratamiento de las maloclusiones de Clase III utilizando la técnica $M E A W$ fue la compensación dentoalveolar, encontrando también, que la mejora en la relación molar y el overjet se logró con el movimiento vertical y distal de los dientes posteriores inferiores ${ }^{24}$. También Won-Sik Yang et al. concluyen en su estudio que los arcos $M E A W$ tienen una relación carga/deflección con un propiedad mecánica única pues permite el movimiento de los dientes individualmente y transmiten la fuerza elástica de manera efectiva a través de todo el alambre ${ }^{25}$.

Por lo tanto, se puede concluir que el tratamiento con la filosofía $M E A W$ es más eficiente para el movimiento distal en masa que el arco ideal simple sin ansas. Si el arco ideal simple sin ansas, no puede verticalizar los molares inferiores, los incisivos inferiores tienen que ser por lo tanto, extruidos e inclinados de manera excesiva hacia lingual para producir una rotación del plano oclusal mandibular en sentido antihorario. Sin embargo, el sistema biomecánico de los arcos $M E A W$ puede distoinclinar efectivamente los molares inferiores y minimizar la extrusión de los incisivos inferiores, por lo que la corrección de una maloclusión de Clase III será relativamente más fácil y con mejores resultados tanto en la posición dentoalveolar como en la estética final del paciente, con la mecánica $M E A W$ que con el arco ideal simple sin ansas.

Sin embargo, en la Figura 10 se puede observar en el control a un ańo de tratamientola aparición de algunos espacios que no habían al culminar el tratamiento. Con lo cual no podemos decir que, en este tratamiento con el 
sistema $M E A W$ se obtuvo estabilidad total en el tiempo, pues se observa cierta recidiva.

\section{Referencias bibliográficas}

1. Sugawara J. Principios de prácticas clínicas para el desarrollo de la maloclusión Clase III. En: Nanda R, editor. Biomecánicas y estética, estrategias en ortodoncia clínica. 1. ${ }^{a}$ ed. Amolca; 2007. p.211-241.

2. Ngan P. Tratamiento de malocclusion de Clase III en dentición primaria y mixta. En: Bishara $S$, editor. Textbook of Orthodontics. 1. ${ }^{\mathrm{a}}$ ed. Philadelphia: Elsevier; 2001.p. 375-411.

3. Zere E, Prabhat Kumar C, Jitendra S, Kunaal D, Nitesh T. Developing Class III malocclusions: challenges and solutions. Clin Cosmet Investig Dent. 2018;10:99-116.

4. Stellzig-Eisenhauer A, Lux C, Schuster G. Treatment decision in adult patients with Class III malocclusion: Orthodontic therapy or orthognathic surgery?. Am J Orthod Dentofacial Orthop. 2002;122:27-38.

5. Ning F, Duan Y. Camouflage treatment in adult skeletal Class III cases by extraction of two lower premolars. Korean J Orthod. 2010;40(5):349-357.

6. Guyer E, McNamara J, Ellis E, Behrents R. Components of class III in juveniles and adolescents. Angle Orthod. 1986;56(1):7-28.

7. Slavicek.R, Sadao Sato. The Masticatory Organ. The dynamic functional anatomy of the craniofacial complex and its relation to the articulation of dentition. J. Stomat. Occ. Med. 2008;1:497-513.

8. Beltrão P. Non-Surgical Treatment of Class III with Multiloop Edgewise Arch-Wire (MEAW) Therapy. En: Virdi MS, editor. Emerging Trends in Oral Health Sciences and Dentistry. Croacia; 2015. p. 443-462.

9. Kim HS, Kim SY. Compensatory changes of a occlusal plane angles in relation to a skeletal factors. Korean J Orthod. 2004;34(3):229-40.

10. Kim YH. A Comparative Cephalometric Study of Class II, Division 1 Nonextraction and Extraction Cases. Angle orthod. 1979;49(2):77-84.

11. Arriola-Guillén LE, Aliaga-Del Castillo A, Pérez-Vargas LF, Flores-Mir C. Influence of maxillary posterior discrepancy on upper molar vertical position and facial vertical dimensions in subjects with or without skeletal open bite. Eu J Orthod. 2015;38(3):251-8.

12. Kim YH. Anterior open bite and its treatment with Multiloop Edge Wise Archwire. Angle orthod.1987;57(4):290-321.

13. Kuo CC, Chen YJ, Lai EHH, Yao CCY, Chang JZC. Long-term stability of an adult Class III open-bite ma- locclusion treated with multiloop edgewise archwire. Journal Dental Sciences. 2009;4(3):149-158.

14. Marańón-Vásquez G, Soldevilla LC, Tolentino FA, Cliff W, Romano FL. Aesthetic and functional outcomes using a multiloop edgewise archwire for camouflage orthodontic treatment of a severe Class III open bite malocclusion. J Orthod. 2017:44(3):1-10.

15. Sato S. Manual for the Clinical Application of MEAW Technique: MEAW; Orthodontic Therapy Using Multiloop Edgewise Arch-wire. Japan: Dental college; 2001.

16. Shirasu A, Sato S. Orthodontic Treatment of Malocclusion (Using the GEAW System). En: Scientific Report Reconstructing a functional occlusion, Japon: Research Institute of Occlusion Medicine, Kanagawa Dental University; 2001. p. 1-16.

17. McLaughlin R, Bennett J, Trevisi H. Revisión del tratamiento de la Clase III. En: McLaudhlin R, editor. Mecánica sistematizada del tratamiento ortodóncico. Madrid: Elsevier; 2002. p. 219-242.

18. Seo YJ, Chung KR, Kim SH, Nelson G. Camouflage treatment of skeletal Class III malocclusion with asymmetry using a bone-borne rapid maxillary expander. Angle Orthodo. 2015;85(2):322-334.

19. Burns NR, Musich DR, Martin C, Razmus T, Gunel E, Ngan P. Class III camouflage treatment: what are the limits?. Am J of Orthod Dentofacial Orthop. 2010;137(1):9.e1-9.e13.

20. Janson G, De Souza JE, Alves FA, Andrade P, Nakamura A, Freitas MR, et al. Extreme dentoalveolar compensation in the treatment of Class III malocclusion. Am J Orthod Dentofacial Orthop. 2005;128(6):787-794.

21. Troy BA, Shanker S, Fields HW, Vig K, Johnston W. Comparison of incisor inclination in patients with Class III malocclusion treated with orthognathic surgery or orthodontic camouflage. Am J Orthod Dentofacial Orthop. 2009;135(2):146.e1-9

22. Sato S. Case report: development characterization of skeletal class III maloclusion. Angle orthod.1994; 64(2):105-10.

23. Chang YLl, Shin SJ, Baek SH. Three-dimensional finite element analysis in distal en masse movement of the maxillary dentition with the multiloop edgewise archwire. Eu J Orthod. 2004; 26(3):339-45.

24. Baek SH, Shin SJ, Ahn SJ, Chang YLl. Initial effect of multiloop edgewise archwire on the mandibular dentition in Class III malocclusion subjects. A three-dimensional finite element study. Eu J Orthod. 2008;30(1):10-5.

25. Yang WS, Kim BH, Kim YH. A Study of the Regional Load Deflection Rate of Multiloop Edgewise Arch Wire. Angle orthod. 2001;71(2):103-9. 